\title{
Bone marrow-derived progenitor cells in pulmonary fibrosis
}

\author{
Naozumi Hashimoto, Hong Jin, Tianju Liu, Stephen W. Chensue, and Sem H. Phan \\ Department of Pathology, University of Michigan, Ann Arbor, Michigan, USA
}

\begin{abstract}
The origin of fibroblasts in pulmonary fibrosis is assumed to be intrapulmonary, but their extrapulmonary origin and especially derivation from bone marrow $(\mathrm{BM})$ progenitor cells has not been ruled out. To examine this possibility directly, adult mice were durably engrafted with BM isolated from transgenic mice expressing enhanced GFP. Induction of pulmonary fibrosis in such chimera mice by endotracheal bleomycin (BLM) injection caused large numbers of $\mathrm{GFP}^{+}$cells to appear in active fibrotic lesions, while only a few $\mathrm{GFP}^{+}$cells could be identified in control lungs. Flow-cytometric analysis of lung cells confirmed the BLM-induced increase in $\mathrm{GFP}^{+}$cells in chimera mice and revealed a significant increase in $\mathrm{GFP}^{+}$cells that also express type I collagen. $\mathrm{GFP}^{+}$lung fibroblasts isolated from chimera mice expressed collagen and telomerase reverse transcriptase but not $\alpha$-smooth muscle actin. Treatment of isolated $\mathrm{GFP}^{+}$fibroblasts with TGF- $\beta$ failed to induce myofibroblast differentiation. Cultured lung fibroblasts expressed the chemokine receptors CXCR4 and CCR7 and responded chemotactically to their cognate ligands, stromal cell-derived factor- $1 \alpha$ and secondary lymphoid chemokine, respectively. Thus the collagen-producing lung fibroblasts in pulmonary fibrosis can also be derived from BM progenitor cells.
\end{abstract}

J. Clin. Invest. 113:243-252 (2004). doi:10.1172/JCI200418847.

\section{Introduction}

Stem cells in the adult have traditionally been thought to be restricted in their potential to differentiate and regenerate tissues in which they reside. However, recent advances have necessitated reconsideration of current knowledge of adult stem cell potential (1). After transplantation of bone marrow (BM) hematopoietic stem cells or nonhematopoietic mesenchymal stem cells, muscle (2), heart (3), liver (4), and lung $(5,6)$ cells of donor origin have been detected. With respect to specific repopulation of lung cells, about $20 \%$ engraftment of type II pneumocytes from a single BM-derived stem cell has been demonstrated (5).

Idiopathic pulmonary fibrosis (IPF) is a devastating disease for which no effective therapy exists $(7,8)$. The hallmark lesions are the fibroblast foci representing focal areas of active fibrogenesis featuring

Received for publication May 6, 2003, and accepted in revised form October 28, 2003.

Address correspondence to: Sem H. Phan, Department of Pathology, University of Michigan Medical School, Ann Arbor, Michigan 48109-0602, USA. Phone: (734) 763-6454;

Fax: (734) 936-1938; E-mail: shphan@umich.edu.

Conflict of interest: The authors have declared that no conflict of interest exists.

Nonstandard abbreviations used: bone marrow (BM); bleomycin (BLM); idiopathic pulmonary fibrosis (IPF); type I collagen (Col I); telomerase reverse transcriptase (TERT); $\alpha$-smooth muscle actin $(\alpha$-SMA); plasma-derived serum (PDS); stromal cell-derived factor- $1 \alpha$ (SDF-1 $\alpha)$; secondary lymphoid chemokine (SLC); insulin, transferrin, and selenium (ITS); phycoerythrin (PE); streptavidin-Cy-Chrome (SAv-Cy-Chrome); streptavidin-CY3 (SAv-Cy3); Tyramide Signal Amplification (TSA); C57BL/6 mice (B6 mice); transgenic (Tg); bone marrow transplantation (BMT); complete medium (CM); lung fibroblasts from BLM-treated B6 mice (BLF); lung fibroblasts from saline-treated B6 mice (SLF). vigorous fibroblast replication and exuberant extracellular matrix deposition, which may lead to obliteration of the distal air space. This has led to a greater focus on fibroblasts and their direct involvement in the fibrotic pathway itself (9).

Fibroblasts represent the key source of interstitial collagens, but these cells are known to be heterogeneous with respect to a number of phenotypic features (10). While it is assumed that they arise from intrapulmonary cells, there is recent evidence of circulating blood cells (termed "fibrocytes") that possess fibroblast-like properties and that can be chemotactically recruited to sites of tissue injury (11). Combined with the recent evidence of BM stem cell plasticity cited above, this provides a compelling argument for reexamination of the origin of the fibroblasts in pulmonary fibrosis, especially in terms of their possible extrapulmonary origin. Such a possibility would have profound implications for our present understanding of pathogenesis and for the development of future novel therapeutic approaches for controlling or managing pulmonary fibrosis.

These considerations led us to investigate whether BM-derived cells could represent a significant source of lung fibroblasts in an animal model of pulmonary fibrosis, and if so, to determine the possible relationship between recruited fibroblasts and the various fibroblast phenotypes that have been described in fibrotic lung lesions. Using BM chimera mice expressing enhanced GFP only in BM-derived cells but not in other tissue cells, we found that substantial numbers of BM-derived fibroblast-like cells migrated to the lung in bleomycin-induced (BLM-induced) pulmonary fibrosis. More than $27 \%$ of the $\mathrm{GFP}^{+}$cells in fibrotic 
lung tissue expressed type I collagen (Col I), more than a fivefold increase over saline-treated control mice. These cells constitute more than $80 \%$ of all Col Iexpressing cells in the fibrotic lung, suggesting that the predominant collagen-producing cell in fibrosis arose from $\mathrm{BM}$ and migrated to the lung in response to signals released in reaction to lung injury and fibrosis. Some of these signals may be chemokines that are expressed in BLM-induced pulmonary fibrosis. Notably, in vitro analysis of $\mathrm{GFP}^{+}$lung fibroblasts cultured from BLM-treated mice revealed that in addition to Col I, these cells also express telomerase reverse transcriptase (TERT) component but not $\alpha$-smooth muscle actin ( $\alpha$-SMA). Thus BM-derived cells seem to give rise to activated fibroblast phenotypes and represent the primary source of Col I expression in BLMinduced pulmonary fibrosis.

\section{Methods}

Materials. DMEM and antibiotics $(100 \mathrm{U} / \mathrm{ml}$ penicillin, $100 \mu \mathrm{g} / \mathrm{ml}$ streptomycin, and $0.25 \mu \mathrm{g} / \mathrm{ml}$ fungizone) were from Invitrogen Corp. (Carlsbad, California, USA). Plasma-derived serum (PDS) was from Cocalico Biologicals Inc. (Reamstown, Pennsylvania, USA). Recombinant human PDGF, recombinant human EGF, recombinant mouse stromal cell-derived factor- $1 \alpha$ (SDF-1 $\alpha$, CXCL12), and recombinant mouse secondary lymphoid chemokine (SLC, CCL21) were from R\&D Systems Inc. (Minneapolis, Minnesota, USA). Insulin, transferring, and selenium (ITS) liquid media supplement, gelatin, and 30\% (w/w) $\mathrm{H}_{2} \mathrm{O}_{2}$ were from Sigma-Aldrich (St. Louis, Missouri, USA). Collagenase type III and DNase were from Worthington Biochemical Corp. (Lakewood, New Jersey, USA). Rabbit biotin-conjugated anti-Col I antibody and goat FITC-conjugated anti GFP antibody were from Rockland (Gilbertsville, Pennsylvania, USA). Mouse biotin-conjugated anti- $\alpha$-SMA antibody (clone 1A4) was from Lab Vision Corp. (Fremont, California, USA). Mouse biotin-conjugated anti-mouse CD45.2 antibody (clone 104), rat phycoerythrin-conjugated (PE-conjugated) anti-mouse Mac-3 antibody (clone M3/84), streptavidin-CyChrome (SAv-Cy-Chrome), Fc block (clone 2.4G2), and BD Cytofix/Cytoperm Kit were from BD Biosciences (San Diego, California, USA). Rat PE-conjugated anti-mouse F4/80 antibody (clone CI:A3-1) was from Caltag Laboratories Inc. (Burlingame, California, USA). Rabbit polyclonal anti-TERT antibody was from Santa Cruz Biotechnology Inc. (Santa Cruz, California, USA). Streptavidin-CY3 (SAv-Cy3) was from Zymed Laboratories Inc. (South San Francisco, California, USA). Histochoice MB was from Amresco Inc. (Solon, Ohio, USA). Tyramide Signal Amplification (TSA) Biotin System was from PerkinElmer (Boston, Massachusetts, USA). The Vector M.O.M. Immunodetection Kit was from Vector Laboratories Inc. (Burlingame, California, USA). BLM was from Faulding Pharmaceutical Co. (Elizabeth, New Jersey, USA).
Superscript One-Step RT-PCR with platinum Taq Kit was from Invitrogen Life Technologies (Carlsbad, California, USA). Costar Transwell was from Corning Costar Corp. (Cambridge, Massachusetts, USA).

Mice. Female 6- to 8-week-old C57BL/6 (B6) mice were purchased from The Jackson Laboratory (Bar Harbor, Maine, USA). GFP-transgenic (Tg) mice on C57BL/6 background were kindly provided by S. Lira (ScheringPlough Research Institute, Kenilworth, New Jersey, USA) (12). All animal studies have been reviewed and approved by the University Committee on Use and Care of Animals at the University of Michigan.

$B M$ chimera mice. BM chimeras were prepared as previously described with minor modification $(13,14)$. BM cells were collected from femurs and tibias of donor GFP Tg or wild-type B6 mice by aspiration and flushing. Recipient B6 mice were exposed to two doses of 5 Gy given 3 hours apart using a ${ }^{137} \mathrm{Cs}$ irradiator, and then maintained on acidified water and autoclaved feed ad libitum. After irradiation, $4 \times 10^{6} \mathrm{BM}$ cells from GFP Tg or B6 mice in a volume of $200 \mu$ l sterile PBS were injected retro-orbitally under anesthesia.

Analysis of organ samples from transplant recipients. To verify successful engraftment and reconstitution of the BM in transplanted mice, BM, peripheral blood, and spleen samples were collected and analyzed at days 28 and 56 after bone marrow transplantation (BMT). Briefly, blood from the retro-orbital vein was collected in PBS with an anticoagulant, and the red blood cells were lysed with a Tris-ammonium chloride buffer. Spleen samples were gently homogenized and depleted of red blood cells as with the blood samples. Femoral BM cells were washed with PBS. The nucleated cells from these samples were then stained with biotin-conjugated anti-mouse CD45.2 antibody which is expressed on all leukocytes of recipient B6 mice, and then detected by subsequent staining with SAv-Cy-Chrome with enumeration by flow cytometry.

Mouse fibrosis model. After durable BM engraftment had been established, pulmonary fibrosis was induced by endotracheal BLM injection as before (15). Briefly, BLM was suspended in sterile saline at $1 \mathrm{U} / \mathrm{ml}$. BM chimera mice were treated with 0.0015 units/g body weight of BLM diluted in sterile saline or the same volume of sterile saline only at day 28 after BMT. The day of the BLM administration was designated as BMT day 28/BLM day 0. Separately where indicated, B6 mice without BM reconstitution were similarly treated with BLM or saline as a source of lung fibroblasts for studies of chemotaxis.

Morphological analysis. At day 28 after BLM injection (BMT day 28/BLM day 28), BM chimera mice were euthanized and both lungs were thoroughly perfused with saline to remove blood from the lung vascular beds as before (15). The lungs were then removed from the thoracic cavity and cleared of extraneous tissue. The lungs were fixed in $4 \%$ paraformaldehyde on ice for 6 hours, dehydrated through graded sucrose washes for 24 hours, and finally fixed in OCT compound 
(Miles Inc., Elkhart, Indiana, USA). Serial cryostat sections ( $4 \mu \mathrm{m}$ thick) were then either stained with $\mathrm{H} \& \mathrm{E}$ or left unstained for fluorescence microscopy to evaluate distribution or localization of $\mathrm{GFP}^{+}$cells.

Analysis of whole-lung cells by flow cytometry. On day 21 after BLM injection (BMT day 28/BLM day 21), lungs from BM chimera mice were removed as above and used to obtain single-cell suspensions for flow-cytometric analysis as previously described (15). Briefly, after mincing, the samples were digested with collagenase and DNase, and then filtered to obtain single-cell suspensions. After appropriate washing and blocking with Fc block, the cells were fixed, permeabilized with BD Cytofix/Cytoperm Kit, stained with the appropriate dilutions of biotin-conjugated anti-Col I antibody or biotin-conjugated isotype-matched control IgG, and detected by subsequent staining with SAv-CyChrome. Some cells were stained with rat PE-conjugated anti-mouse F4/80 antibody, rat PE-conjugated anti-mouse Mac-3 antibody, or rat PE-conjugated isotype-matched control IgG before fixation. Dead cells and erythrocytes were excluded from analysis by appropriate gating. Flow-cytometric analysis was undertaken using an Epics XL-MCL machine (Beckman Coulter Inc., Miami, Florida, USA). Data collected were analyzed using Winlist software (Verity Software House Inc., Topsham, Maine, USA).

Mouse lung fibroblast culture. Mouse lung fibroblasts were isolated from lung tissue by mincing and enzymatic digestion as previously described (15). After filtration, released cells were centrifuged, washed, and cultured in complete medium (CM) composed of DMEM supplemented with $10 \%$ PDS, human recombinant PDGF $(5 \mathrm{ng} / \mathrm{ml})$, recombinant human EGF (10 $\mathrm{ng} / \mathrm{ml}$ ), ITS liquid media supplement (1:100), and antibiotics. The cells were maintained in culture and passaged as before (15). Fibroblasts in this study were used after the second passage.

Where indicated, fibroblast monolayers were treated with $10 \mathrm{ng} / \mathrm{ml} \mathrm{TGF}-\beta$ for 24 hours and then harvested for assessment of GFP mRNA by RT-PCR (see below) or $\alpha$-SMA expression by flow cytometry. The expression of $\alpha$-SMA was analyzed by flow cytometry using the same protocol as for the analysis of Col I in whole-lung cells. Immunocytochemistry for cultured fibroblasts. Immunostaining procedures were done as previously described with some modification using the TSA Biotin System (16). Unless otherwise specified, incubation and wash procedures were routinely carried out at room temperature. For analysis of cultured fibroblasts, the cells were suspended in CM and plated at $1 \times 10^{5}$ cells $/ \mathrm{ml}$ into an eight-well Lab-Tek Chamber Slide System (Nalge Nunc International, Naperville, Illinois, USA). After 24 hours, the slides were air-dried and fixed in Histochoice MB solution for 20 minutes. Endogenous peroxidase activity was inactivated by $0.3 \% \mathrm{H}_{2} \mathrm{O}_{2}$ in methyl alcohol for 30 minutes, and after washing, the slides were immersed in $0.2 \%$ Triton X-100 in PBS for 15 minutes. After blocking nonspecific protein binding with the blocking buffer (supplied in the TSA Biotin System Kit), the slides were incubated overnight at $4{ }^{\circ} \mathrm{C}$ with appropriate dilutions of rabbit biotin-conjugated anti-Col I antibody, mouse biotin-conjugated anti- $\alpha$-SMA antibody, or rabbit antiTERT antibody. As negative controls, each primary antibody was substituted with the appropriate species- and isotype-matched immunoglobulins. When mouse monoclonal antibody was used, the Vector M.O.M. Immunodetection Kit was used according to the manufacturer's recommendation. For TERT immunostaining, a secondary goat biotinylated anti-rabbit IgG was used. All slides were sequentially incubated with streptavidin-peroxidase complex, biotinyl tyramide, and SAv-Cy3, with washes in between each incubation. GFP expression was detected with goat FITC-conjugated anti-GFP antibody. The sections were mounted with Immu mount (Shandon Lipshaw Inc., Pittsburgh, Pennsylvania, USA). A minimum of five randomly selected high-power fields were examined per sample to count cells expressing the antigen of interest until at least a total cell count of 100 was reached. Cells positive for Col I, $\alpha$-SMA, or TERT in $\mathrm{GFP}^{+}$and $\mathrm{GFP}^{-}$cells were counted and expressed as a percentage of total cells counted for each sample. A total of five samples were examined (17).

PCR analysis for expression of GFP, chemokines, and chemokine receptors. Total lung RNA was isolated from lung tissue or isolated lung fibroblasts as previously described (15). Semiquantitative PCR analysis was done as previously described (18). Real-time PCR was performed on a TaqMan ABI 5700 Sequence Detection System (PE Biosystems, Foster City, California, USA). Using TaqMan one-step RT-PCR master mix reagents, $100 \mathrm{ng}$ of isolated total RNA was reverse transcribed at $48^{\circ} \mathrm{C}$ for 30 minutes, and after serial denaturation at $95^{\circ} \mathrm{C}$ for 10 minutes, cDNA was amplified as follows: 50 cycles of a two-step PCR program at $95^{\circ} \mathrm{C}$ for 15 seconds and $60^{\circ} \mathrm{C}$ for 60 seconds. The following oligonucleotide primers $(200 \mathrm{nM})$ and probes $(200 \mathrm{nM})$ were used: mouse SDF-1 $\alpha$ (GenBank accession number L12029; bp 347-417) sense (5'-AGTAAGCACAACAGCCCAAAGG-3') and antisense (5'CTTGCATCTCCCACGGATGT- $3^{\prime}$ ), internal fluorescencelabeled probe (FAM) (5'-TTCCAGTAGACCCCCGAGGAAGGC-3'); mouse SLC/CCL21 (GenBank accession number NM_011335; bp 518-586) sense (5'-CAGGCAAAGAGGGAGCTAGAAA- ${ }^{\prime}$ ) and antisense (5'-TGGACGGAGGCCAGCAT- $3^{\prime}$ ), and internal probe (FAM) (5'-TCAGGAGCCCAAAGCAGCCACC- $3^{\prime}$ ). These primers and probes were obtained from PE Biosystems. The mRNA levels were normalized to GAPDH mRNA signal using TaqMan rodent GAPDH control reagents (19). In preliminary experiments, we confirmed that the PCR products, which were pre-formed using the target gene-specific primers, were not contaminated with fragments amplified from genomic DNA by comparing the products generated with and without reverse transcriptase.

RT-PCR analysis for mRNA of GFP and chemokine receptors, CXCR4 and CCR7, was performed using Superscript One-Step RT-PCR with platinum Taq kit. Primer sequences used for GFP and chemokine receptors 
were as follows: enhanced GFP (475-bp product), sense (5'-AAGTTCATCTGCACCACCG-3'), antisense (5'-TGCTCAGGTAGTGGTTGTCG-3') (20); CXCR4 (480-bp product), sense ( $5^{\prime}$-ACCATCTACTTCATCATCTTC-3'), antisense (5'-CACCATCCACAGGCTATC-3'); CCR7 (586-bp product), sense ( $5^{\prime}$-TGGTGGTGGCTCTCCTTGTC- $\left.3^{\prime}\right)$, antisense $\left(5^{\prime}\right.$-TCCTCGCCGCTGTTCTTCTG- $\left.3^{\prime}\right)$. For GAPDH (308-bp product), the primers were sense ( $5^{\prime}$-GCAGTGGCAAAGTGGAGATT-3') and antisense (5'-GCAGAAGGGGCGGAGATGAT-3') (21). As positive control, RNA samples from spleen were used. Intensity of PCR products were measured by Kodak 1D image analysis software (Kodak Scientific Imaging Systems, New Haven, Connecticut, USA). The mRNA levels of CXCR4 and CCR7 were normalized to the signal for GAPDH.

Fibroblast chemotaxis assay. Chemotaxis assays were performed using Costar Transwell inserts ( $8-\mu \mathrm{m}$ pore size), which were precoated with $0.1 \%$ gelatin, essentially as previously described $(11,22,23)$. Isolated murine lung fibroblasts were suspended at $1 \times 10^{6}$ cells/ml in DMEM containing $0.1 \%$ BSA. Medium alone (negative control) or medium containing SLC or SDF- $1 \alpha(600 \mu \mathrm{l})$ at the indicated concentration was added to individual wells of a 24-well plate. Transwell devices were then inserted, and the fibroblasts $(100 \mu \mathrm{l})$ were layered on top of the membrane of the upper chamber of the Transwell insert (three wells per condition) and incubated at $37^{\circ} \mathrm{C}$ in a moist $5 \% \mathrm{CO}_{2} / 95 \%$ air atmosphere. After 4 hours, the cells on the top of the filter were removed by scraping. The filter was fixed with methanol for 10 minutes, and then stained with Hematoxylin QS (Vector Laboratories Inc.). For checkerboard analysis of SLC- or SDF-1 $\alpha-$ directed chemotaxis of fibroblasts, SLC at $250 \mathrm{ng} / \mathrm{ml}$ or SDF- $1 \alpha$ at $500 \mathrm{ng} / \mathrm{ml}$ was added to both the bottom and top chambers. Migration was assessed by counting the number of cells in five high-power fields with a light microscope. Replicate experiments were performed with separate cultures of cells on separate occasions.

Statistical analysis. The results were analyzed using the Mann-Whitney test for comparison between any two groups, and by nonparametric equivalents of ANOVA for multiple comparisons. $P<0.05$ was considered to indicate statistical significance.

\section{Results}

Creation of GFP BM chimera mice. Successful transplantation requires adequate extirpation of the recipient $\mathrm{BM}$ followed by survival and engraftment of the trans- planted BM cells. In this BMT protocol, irradiated recipient B6 mice without BMT (injected with PBS only) died within 21 days. To verify whether durable BM engraftment had been established, we examined the extent to which CD $45.2^{+}$cells were also positive for GFP in BM, peripheral blood, and spleen by flow cytometry at days 28 and 56 after BMT. As shown in Figure 1 , on day 28 , more than $92 \%$ of CD $45.2^{+}$cells in $\mathrm{BM}$ and blood were $\mathrm{GFP}^{+}$, while in the spleen more than $77 \%$ were $\mathrm{GFP}^{+}$. These percentages of $\mathrm{GFP}^{+}$cells remained essentially unchanged by day 56 after BMT in both BM and peripheral blood, while the level increased to more than $88 \%$ in the spleen. These data indicated that the recipient $\mathrm{B} 6$ mice had been adequately irradiated, and completely as well as durably reconstituted with BM cells of GFP Tg mouse origin.

We gave recipient $\mathrm{B} 6$ mice two doses of 5 Gy each given 3 hours apart to achieve the maximum effect for $\mathrm{BM}$ reconstitution while minimizing the undesirable effects of irradiation (e.g., to the lungs and other vital tissues). Since radiation may induce pneumonitis that may influence the endpoints to be studied in the BLM model, we first evaluated the effect of irradiation on the lungs of GFP BM chimera mice without BLM treatment. Histological examination of H\&E-stained lung sections revealed essentially normal lung architecture without evidence of inflammation or fibrosis at day 28 after BMT (Figure 2a). Similar evaluation on day 56 after BMT revealed no significant change from day 28 , although a few scattered inflammatory cells could now be discerned (Figure $2 \mathrm{~b}$ ). These findings of essentially normal lung in chimera mice were supported by the findings of fluorescence microscopy to evaluate presence and localization of $\mathrm{GFP}^{+}$cells in lungs of these mice. These showed few $\mathrm{GFP}^{+}$cells in serial sections of the lung, even at day 56 after BMT (Figure 2, c and d). Although the histological findings in lungs of recipient B6 mice transplanted with wild-type B6 BM cells were similar to those of GFP chimera mice, they did not show any $\mathrm{GFP}^{+}$cells in the lung under fluorescence microscopy (data not shown). Thus, BMT in the process of creating BM chimera mice had no significant effects on lung morphology.

$B M$-derived $\mathrm{GFP}^{+}$cells in BLM-induced lung fibrosis. To evaluate the influx of $\mathrm{GFP}^{+}$cells into the lungs in BLMinduced fibrosis, we first evaluated the lung histology of chimera mice on day 28 after BLM treatment (i.e., BMT day 28/BLM day 28). The results showed that

\section{Table 1}

Summary of phenotypes in whole-lung cells of BLM- or saline-treated GFP BM chimera mice by flow cytometry ${ }^{\mathrm{A}}$

\begin{tabular}{|c|c|c|c|c|c|}
\hline \multicolumn{6}{|c|}{ Percentage of cells that are: } \\
\hline Treatment & $\mathrm{GFP}^{+}$ & $\mathrm{GFP}^{+} / \mathrm{Col} \mathrm{I}^{+}$ & GFP-/Col I+ & GFP-/Col I- & $\mathrm{GFP}^{+} / \mathrm{Col} \mathrm{I}^{-}$ \\
\hline BLM & $71.6 \pm 6.50$ & $27.5 \pm 8.36$ & $6.63 \pm 0.99$ & $23.8 \pm 5.86$ & $41.9 \pm 4.91$ \\
\hline Saline & $16.5 \pm 6.97$ & $4.76 \pm 2.52$ & $4.83 \pm 3.06$ & $78.7 \pm 6.77$ & $11.7 \pm 5.04$ \\
\hline
\end{tabular}

${ }^{A}$ Data are shown as the percentage of cells that are either positive $(+)$ and/or negative $(-)$ for GFP or Col I in whole-lung cells. Data shown represent the means \pm SD from eight BLM- or saline-treated GFP BM chimera mice, respectively, and are representative of two independent experiments. 


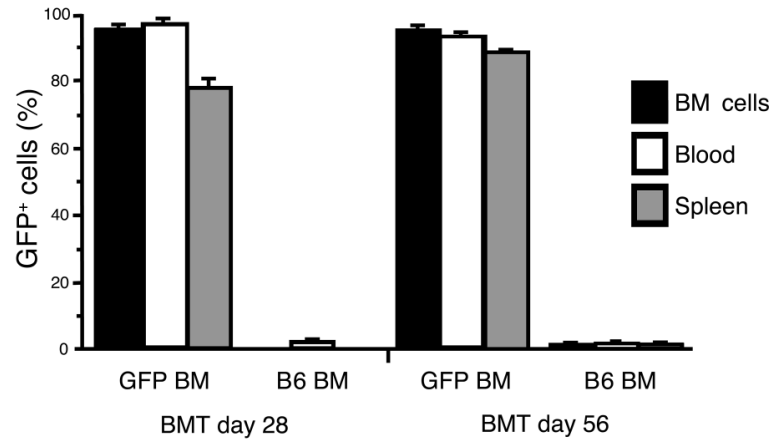

Figure 1

Kinetics of engraftment of GFP+ BM cells. BM, peripheral blood, and spleen from recipient mice were collected at days 28 and 56 after transplantation with BM from GFP Tg mice. The nucleated cells from these samples were immunostained with biotin-conjugated antimouse CD45.2 and then detected by subsequent staining with SAv$\mathrm{Cy}$-Chrome. The percentage of $\mathrm{GFP}^{+}$cells among $\mathrm{CD} 45.2^{+}$cells in these samples were determined by flow cytometry. Data shown represent the means \pm SEM from groups of 6-12 BM chimera mice.

lungs from BLM-treated GFP BM chimera mice exhibited severe pulmonary fibrosis, characterized by loss of normal alveolar architecture, prominent disorganized thickening of the alveolar septa, and collapse of the alveolar space by organizing inflammatory infiltrate and fibroblasts (Figure 3, a and b). These histological changes of lungs from BLM-treated GFP BM chimera mice were comparable to those in BLM-treated B6 mice transplanted with wild-type B6 BM (data not shown). When the same lung tissues from GFP BM chimera mice were analyzed by fluorescence microscopy, large numbers of the cellular elements were found to express GFP, especially densely clustered in cellular areas of active fibrosis (Figure 3c). In contrast, lungs from saline-treated GFP BM chimera mice showed essentially normal lung architecture with a few scattered inflammatory cells visible (Figure $3, \mathrm{~d}$ and e) and very few $\mathrm{GFP}^{+}$cells evident by fluorescence microscopy (Figure 3f). Thus significant numbers of the cells in active fibrotic lesions were derived from the BM.

Phenotype of BM-derived cells in fibrotic lung. Inflammatory/immune cells would be expected to be part of the infiltrating $\mathrm{GFP}^{+}$cells in BLM-treated chimera mice. To evaluate the possibility that additional cell types may also be derived from the BM, whole-lung cells were isolated from BLM- or saline-treated GFP BM chimera mice at BMT day 28/BLM day 21 and then analyzed by flow cytometry (Figure 4). Specifically, the population of cells producing $\mathrm{Col} \mathrm{I}$ in whole-lung cells was examined, since the deposition of Col I is a key characteristic finding in fibrotic lungs. The results confirmed the marked increased in the number of $\mathrm{GFP}^{+}$cells in lungs of BLM-treated chimera mice, with more than $71 \%$ of all cells being BM-derived as opposed to less than $17 \%$ in saline-treated controls (Table 1). As expected, there was more than a threefold increase in the percentage of Col I+ lung cells in BLM-treated chimera mice relative to saline-treated chimera mice, although more than $60 \%$ of the $\mathrm{GFP}^{+}$cells did not express Col I (Table 1 and Figure 4). Surprisingly, however, more than $80 \%$ of the $\mathrm{Col} \mathrm{I}^{+}$cells were $\mathrm{GFP}^{+}$in BLM-treated GFP BM chimera mice, which represented $27.5 \%$ of the whole-lung cells analyzed. In contrast, only $4.76 \%$ of all cells were $\mathrm{Col} \mathrm{I}^{+}$ and $\mathrm{GFP}^{+}$in saline-treated controls. These data demonstrated that BM-derived cells represent the bulk of the Col I-producing cells in BLM-induced lung fibrosis and thus probably play a major role in fibrogenesis. To rule out the contribution of $\mathrm{BM}$-derived macrophages that could have taken up degraded collagen, the population of cells expressing F4/80 or Mac-3, which are both specific markers for macrophages, was also evaluated in $\mathrm{GFP}^{+}$whole-lung cells. Only $6.7 \%$ of the $\mathrm{Col} \mathrm{I}{ }^{+}$ cells, which represented $2.7 \% \pm 0.76 \%$ of $\mathrm{GFP}^{+}$cells analyzed, expressed F4/80 (Figure 4e), while $14.5 \%$ of Col I ${ }^{+}$ cells, representing $5.5 \% \pm 0.4 \%$ of $\mathrm{GFP}^{+}$cells, expressed Mac-3 (Figure 4f). Thus, at most less than $15 \%$ of Col I ${ }^{+}$ cells could be identified as macrophages that may have taken up degraded collagen.

To assess further the phenotype of these BM-derived Col I-expressing cells with respect to their potential identity with previously identified fibroblast phenotypes, lung fibroblasts were isolated for additional in vitro studies. A majority of the lung fibroblasts isolated from BLM-treated GFP BM chimera mice were $\mathrm{GFP}^{+}$and displayed normal, primarily spindle-shaped fibroblast-like
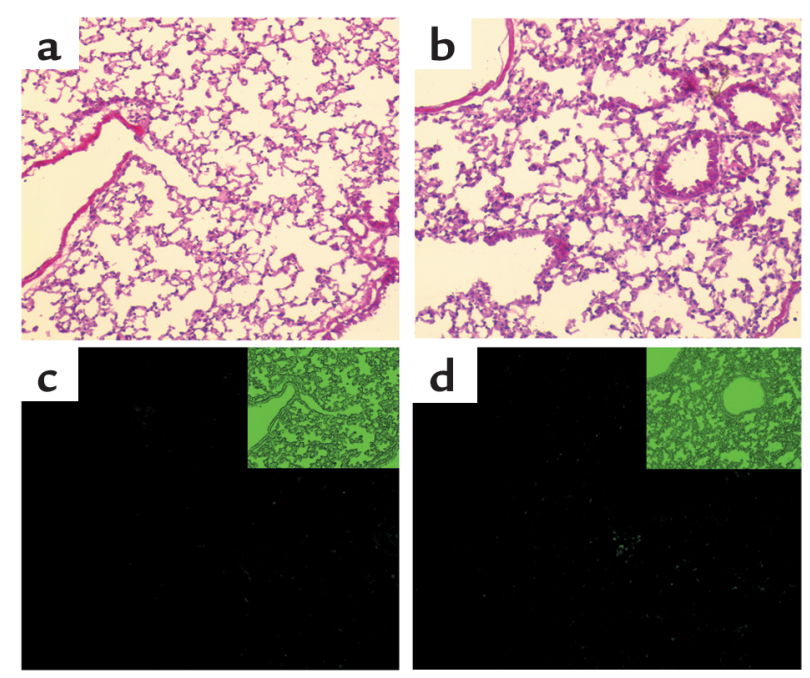

\section{Figure 2}

Lung morphology of BM chimera mice. Representative lung sections from GFP BM chimera mice were examined on day 28 (a and c) and day 56 (b and d) after BMT, by light microscopy (a and $\mathbf{b}$, H\&E-stained sections) and fluorescence microscopy (c and d). At day 28 after BMT (a), lungs from GFP BM chimera mice revealed essentially normal lung architecture and no evidence of pneumonitis. At day 56 after BMT (b), the lungs also appeared essentially normal, except for a few scattered inflammatory cells. Only a few $\mathrm{GFP}^{+}$cells were present in lung sections on both days 28 (c) and 56 (d) after BMT. Insets in $\mathbf{c}$ and $\mathbf{d}$ showed light-microscopic images of the unstained sections used for fluorescence microscopy. All images were photographed at $\times 100$. 

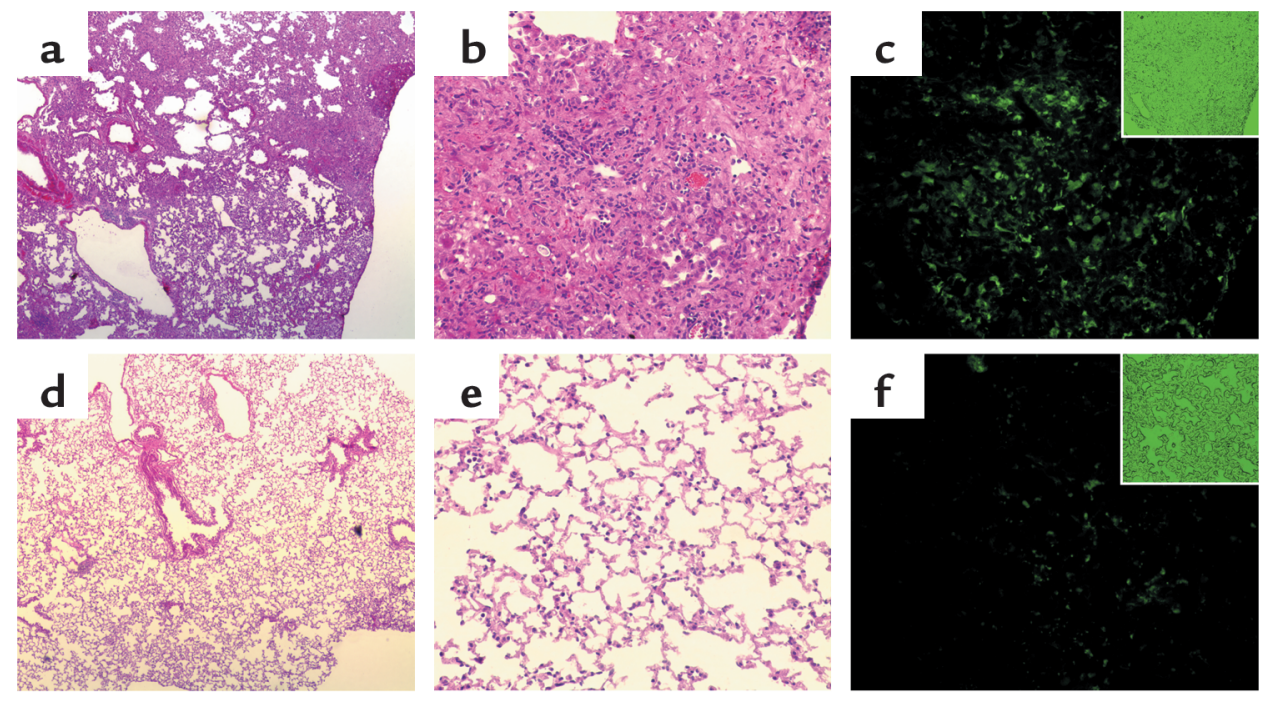

\begin{abstract}
Figure 3
Morphology of BLM-induced lung fibrosis in BM chimera mice. Representative lung sections from BLM-treated (a-c) or saline-treated (d-f) GFP BM chimera mice were evaluated at day 28 after BLM or saline treatment. The H\&E-stained sections revealed severe distortion of lung alveolar architecture due to extensive fibrosis ( $\mathbf{a}$ and $\mathbf{b}$, at $\times 40$ and $\times 200$, respectively). (c) Numerous GFP cells in densely cellular fibrotic lesions were easily identifiable by fluorescence microscopy in BLM-treated lungs. $\times 200$. In contrast, H\&E-stained lung sections from salinetreated GFP BM chimera mice showed normal lung architecture ( $\mathbf{d}$ and $\mathbf{e}$, at $\times 40$ and $\times 200$, respectively), with a few scattered GFP+ cells visualized by fluorescence microscopy $(\mathbf{f}$, at $\times 200)$. Insets in $\mathbf{c}$ and $\mathbf{f}$ showed the light-microscopic appearance of the respective sections examined by fluorescence microscopy. $\times 200$.
\end{abstract}

morphology (Figure 5a). The distribution of the positive immunostaining for GFP (green) in cultured cells was consistent with that of the intrinsic GFP fluorescence seen without immunostaining (Figure $5 \mathrm{~b}$ ). All of the cultured cells stained positively for Col I (red) intracellularly (Figure 5c). Double immunostaining for GFP (green) and $\mathrm{Col} I$ (red) revealed that virtually all of these $\mathrm{GFP}^{+}$ fibroblast-like cells express Col I (appears yellow in Figure $5 \mathrm{~d}$ ), of which fibroblasts are known to be a major producer (24). These results confirmed that BM-derived fibroblast-like cells could directly contribute to the deposition of collagen in pulmonary fibrosis.

To determine whether any of these BM-derived cells were myofibroblasts, the cells were also immunostained for $\alpha$-SMA, a marker of differentiated myofibroblasts (25). Some of the cells showed the morphological characteristics of myofibroblasts and stained positively (red) for $\alpha$-SMA (Figure 5e). However, by dual immunofluorescence, the positive staining for $\alpha$-SMA was seen primarily in cells that were negative or showing only background staining for GFP (Figure 5e). These findings suggested that most, if not all, myofibroblasts were not derived from BM-derived fibroblastlike cells in this model of pulmonary fibrosis.

Another fibroblast phenotype that has been recently described to be present in pulmonary fibrosis is the expression of TERT (17), a key component of telomerase. To determine if TERT-expressing fibroblasts could be derived from the $\mathrm{GFP}^{+}$cells, we also undertook immunostaining for TERT. Double immunostaining for GFP and TERT showed that a majority of these cells were also $\mathrm{GFP}^{+}$(Figure $5 \mathrm{f}$ ). This was confirmed by counting the cells expressing either antigen, revealing that the percentages of TERT ${ }^{+}$cells among $\mathrm{GFP}^{+}$cells and among total cells were $66 \%$ and $64 \%$, respectively. Thus, a significant portion of the TERT-expressing fibroblast phenotype in BLM-induced pulmonary fibrosis was derived from BM precursor cells.

Effects of TGF- $\beta$ on BM-derived lung fibroblasts. Doubleimmunofluorescence analysis for $\alpha$-SMA and GFP expression suggested that lung myofibroblasts in this model did not arise from BM progenitor cells (Figure 5 e). This would suggest that these cells may be resistant to TGF- $\beta$, a well-known and potent inducer of $\alpha$-SMA expression in fibroblasts and of myofibroblast differentiation (24-26). To evaluate this possibility, cultured lung fibroblasts from BLM-treated GFP chimera mice were incubated with or without TGF- $\beta$, and then analyzed for $\alpha$-SMA expression by flow cytometry. The results showed that less than $1 \%(0.6 \% \pm 0.1 \%)$ of $\mathrm{GFP}^{+}$cells (i.e., BM-derived) expressed $\alpha$-SMA, and this result did not change when the cells were treated with TGF- $\beta(0.40 \% \pm 0.1 \%)$. In contrast, similar treatment of naive lung fibroblasts from B6 mice under identical conditions showed almost a doubling in the proportion of cells expressing $\alpha$-SMA (from $11.4 \% \pm 2.3 \%$ to $19.1 \% \pm 3.1 \%)$. To rule out the possibility that TGF- $\beta$ could downregulate the expression of GFP, cultured lung fibroblasts from GFP Tg mice were incubated with or without TGF- $\beta$ in vitro, and then GFP expression was evaluated by RT-PCR. The results showed that TGF- $\beta$ treatment had no significant effect on GFP expression (data not shown), thus ruling out this possibility as the reason for failure to detect $\alpha$-SMA 
a

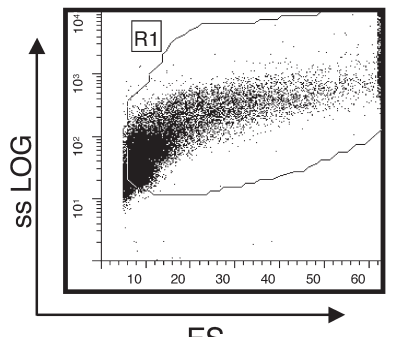

c

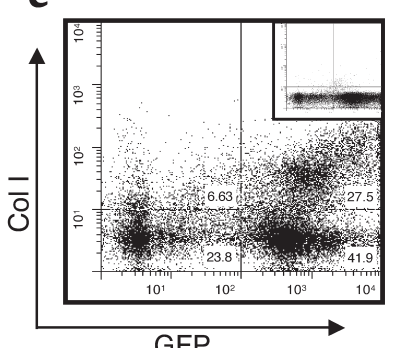

e

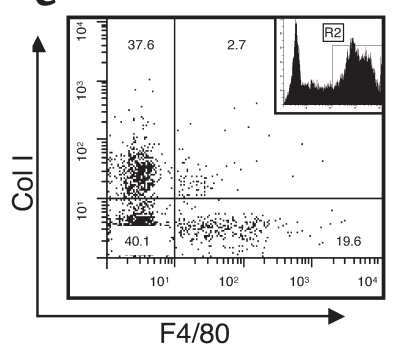

b

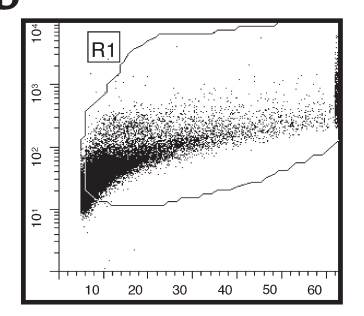

d

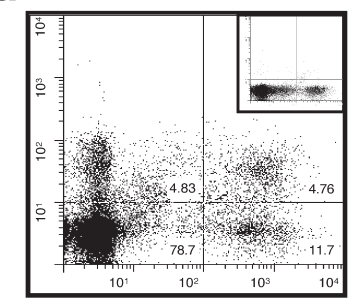

$f$

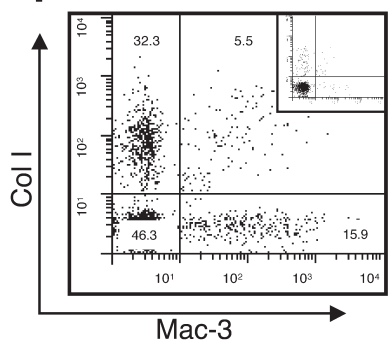

expression in the BM-derived fibroblasts. These results confirmed that the bulk, if not all, of the lung myofibroblasts induced in this model did not appear to arise from BM-derived progenitor cells.

Expression of chemokines and chemokine receptors. BMderived inflammatory cells are known to migrate into BLM-induced fibrotic lesions in the lungs, partly as a result of the secretion of chemokines to which they respond chemotactically. There is evidence also that peripheral blood-derived fibroblasts (fibrocytes) can express several chemokine receptors, especially CXCR4 and CCR7, and respond chemotactically to their respective ligands (11). To determine whether similar mechanisms were at work in recruitment of BMderived precursor fibroblasts to the lung in BLMinduced injury, we first examined the expression in the lung of SDF-1 $\alpha$ and SLC, which are the ligands for CXCR4 and CCR7, respectively. The results showed that SDF- $1 \alpha$ mRNA was detectable in the lungs of untreated mice (Figure 6a). At day 7 after BLM or saline treatment, the SDF- $1 \alpha$ mRNA level in BLMtreated lungs was significantly increased compared with that in saline-treated lungs (4-fold vs. 1.6-fold increase above untreated control lungs, respectively). This elevated expression of SDF- $1 \alpha$ in BLM-treated murine lungs was maintained at day 14 after the treatment (3.5-fold vs. 1.9-fold increase above untreated lungs in saline-treated mice). While lung SLC mRNA

\section{Figure 4}

Flow-cytometric analysis of whole-lung cells from GFP BM chimera mice. Whole-lung cells were isolated from BLM-treated (a and $\mathbf{c}$ ) or saline-treated (b and d) GFP BM chimera mice at day 21 after BLM or saline treatment. Following the appropriate immunostaining, the cells were analyzed by flow cytometry for GFP and Col I expression (c and $\mathbf{d}$ ) after gating on intact live cells (region indicated by R1) according to side scatter (shown in logarithmic scale, ssLOG) and forward scatter (FS) (a and $\mathbf{b}$ ). Results of analysis of cells treated with isotypematched control IgG for the anti-Col I antibody are shown in the insets in $\mathbf{c}$ and $\mathbf{d}$. Furthermore, the $\mathrm{GFP}^{+}$cells from these BLM-treated mice were analyzed for Col I and F4/80 or Mac-3 expression after gating on $\mathrm{GFP}^{+}$cells in the $\mathrm{R} 1$ region (region indicated by $\mathrm{R} 2$ in the inset in e) (e and $\mathbf{f})$. The Col $\mathrm{I}^{+}$and $\mathrm{F} 4 / 80^{+}$cells represented $2.7 \% \pm 0.76 \%$ of $\mathrm{GFP}^{+}$cells $(\mathbf{e})$. The $\mathrm{Col} \mathrm{I}^{+}$and $\mathrm{Mac}-3^{+}$cells represented $5.5 \% \pm 0.4 \%$ of $\mathrm{GFP}^{+}$cells $(\mathbf{f})$. Inset in $\mathbf{f}$ shows the cells stained with isotype-matched control IgG. Representative runs are shown for each group from a total of eight BLM-treated or saline-treated GFP BM chimera mice, respectively. The quantitative results are summarized in Table 1.

was also significantly increased in BLM-treated murine lungs relative to that in saline controls, the kinetics were different, with the increase not evident until day 14 after BLM treatment (Figure 6b). At day 7 there were no significant differences in the lung expression of SLC between saline- and BLM-treated mice - results that were comparable to those in untreated mice. Thus either of these chemokines could potentially play a role in recruitment of BMderived precursor fibroblasts to the BLM-injured lung.

Analysis of CXCR4 and CCR7 expression was undertaken next to confirm that the receptors for these chemokines are expressed by lung fibroblasts to allow them to respond chemotactically. The results (Figure 6, $\mathrm{c}$ and d) showed that the level of CXCR4 mRNA in lung fibroblasts from BLM-treated B6 mice (BLF) was over twice as high as that from saline-treated B6 mice (SLF). While CCR7 mRNA in BLF was also elevated, the increase was more than sixfold above that in SLF. To confirm that these expressed receptors were actually functional, in vitro chemotaxis assays were undertaken. As shown in Figure 6d, BLF could migrate to both SDF-1 $\alpha$ (CXCR4 ligand) and SLC (CCR7 ligand). The dose of SLC was comparable to that previously shown to be active against fibrocytes (11). Checkerboard analysis confirmed that the migration of fibroblasts was due to a chemotactic response to these chemokines, although SDF- $1 \alpha$ did have minor chemokinetic activity as well. Thus BLM-injured lung tissue showed increased expression of chemokines that had chemotactic activity for lung fibroblasts expressing their cognate receptors.

\section{Discussion}

The potential extrapulmonary origin of lung fibroblasts in pulmonary fibrosis merits investigation because of recent evidence suggesting that pluripotent precursor cells in BM can repopulate distal organs (1-6) and that circulating fibrocytes can migrate to wound-healing sites to serve as a source for 

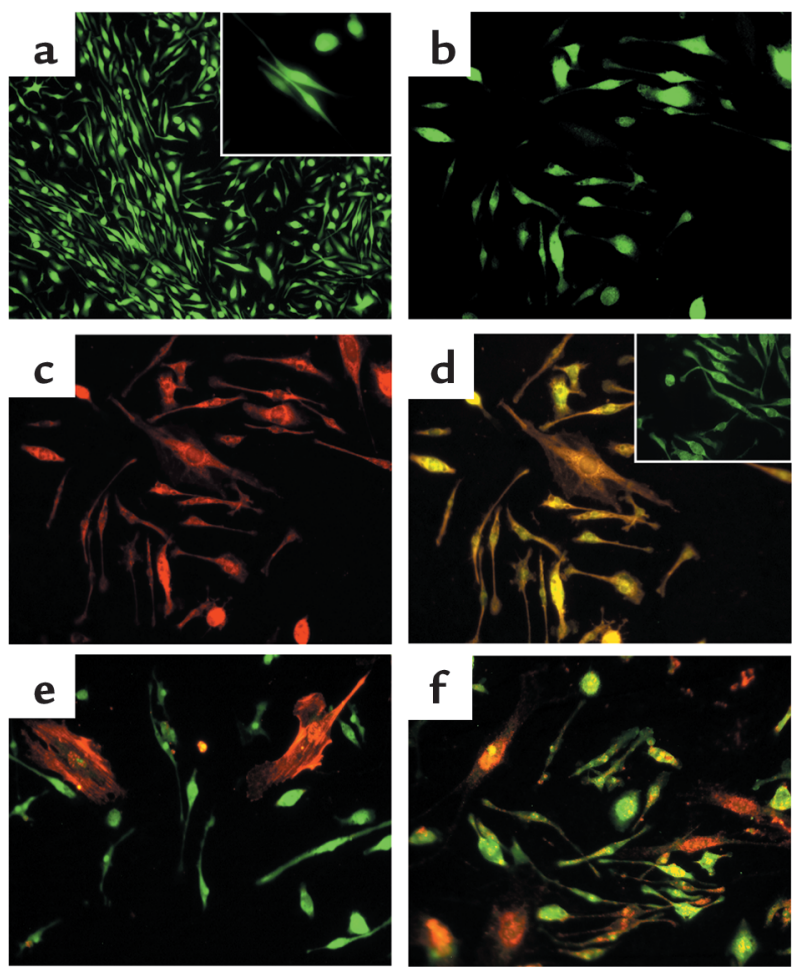

fibroblasts and myofibroblasts that normally participate in the repair process (11). In this study, the possibility that BM-derived precursor cells could serve as a source for fibroblasts in BLM-induced pulmonary fibrosis was examined. While there is no completely satisfactory animal model of human IPF, the BLMinduced model is relatively well characterized and does exhibit certain features found in the human disease. Nevertheless, there are clear limitations to this model in terms of its self-limiting nature, the rapidity of its development, and the close association with inflammation that accompanies the lung injury (15, 17). However the model remains useful for identification of mechanisms and pathogenetic clues that may be relevant to the human disease. Finding such clues may be helpful in providing the basis for human studies to confirm their relevance.

To distinguish clearly the potential role of BM-derived cells in the establishment of fibrosis from that of resident intrapulmonary fibroblasts, we devised a BMT model in which GFP Tg mice were the source of donor BM cells (12). Thus all BM-derived cells in the GFP BM chimera mice could be easily distinguished from resident lung cells by their green fluorescence due to GFP expression. One concern with this approach is the use of a lethal dose of radiation to extirpate the recipient's $\mathrm{BM}$ prior to injection of donor BM. Although the irradiation could cause lung injury and pneumonitis (26-28), we have carefully titered down the radiation dose and, as recommended for $\operatorname{BMT}(13,14)$, divide the total dose into two doses given 3 hours apart. This reduced total dose, and the splitting thereof into two equal doses, caused no respiratory distress, detectable

\section{Figure 5}

Characterization of cultured lung fibroblasts. Lung fibroblasts isolated from BLM-treated GFP BM chimera mice were analyzed by fluorescence microscopy $(\mathbf{a}-\mathbf{f})$. The cells showed typical fibroblast morphology, many being stellate or spindle-shaped (a). An average $80 \%$ of these cells expressed GFP (green fluorescence in $\mathbf{a}$, at $\times 100$; inset at $\times 400$ ). Cells were stained with both anti-GFP (green) and anti-Col I (red) antibodies in $\mathbf{b}-\mathbf{d}$. The same microscopic field was photographed with the green $(\mathbf{b})$ or red (c) filter only, or both simultaneously (d). Colocalization of both GFP and Col I expression resulted in a yellow color in $\mathbf{d}$. Inset in $\mathbf{d}$ shows the cells stained with anti-GFP antibody (green) and isotype-matched control IgG for Col I (red). Cells were also stained with both anti-GFP (green) and anti- $\alpha$-SMA (red) antibodies (e). Colocalization of GFP and $\alpha$-SMA should appear yellow, but the two $\alpha-\mathrm{SMA}^{+}$cells in this field did not appear to express $\operatorname{GFP}(\mathbf{e})$. Finally, cells were also stained with anti-GFP (green) and antiTERT (red) antibodies. Colocalization of GFP and TERT appeared yellow, and most of the cells in this field expressed both TERT and GFP (f). Magnification was $\times 200$ for $\mathbf{b}-\mathbf{f}$. A representative example of at least three independent experiments is shown.

lung injury, or fibrosis, and the mice exhibited normal body weight gain and activity. Consistent with previous studies $(29,30)$, successful, complete, and durable BM reconstitution was achieved using this protocol without evidence of lung pathology up to day 56 after BMT.

Fluorescence-microscopic analysis of normal lung tissue in these chimera mice revealed only a few cells that were $\mathrm{GFP}^{+}$, with intravascular localization that would be consistent for leukocytes, as well as those with epithelial distribution consistent with alveolar pneumocytes. Repopulation of the alveolar epithelium by BM-derived precursor cells has been previously reported (31). In contrast, lungs from BLM-injured chimera mice exhibited vastly increased numbers of cells expressing GFP, virtually all clustered in areas undergoing active fibrosis. A significant portion of these $\mathrm{GFP}^{+}$cells probably represent infiltrating leukocytes, as previously noted in this model (32). At later stages with diminished inflammation (15), fluorescence microscopy revealed considerable numbers of $\mathrm{GFP}^{+}$cells distributed primarily in cellular areas undergoing active fibrosis. The extravascular and interstitial localization of some of these cells, as well as their morphology, were consistent with fibroblasts. Flow-cytometric analysis of the disaggregated cells from lung tissue confirmed more than a fourfold increase in $\mathrm{GFP}^{+}$cells in BLM-injured versus intact lungs, and $27.5 \%$ of cells in fibrotic lung were $\mathrm{GFP}^{+}$and expressed Col I, a marker for fibroblasts (33). In contrast more than $5 \%$ of cells expressed GFP and Col I in control lungs. Thus the increased collagen expression in fibrotic lung was due mainly to BM-derived precursor cells a result that argues for a major direct role for these cells in the pathogenesis of fibrosis.

Additional phenotypic analysis of these BM-derived cells in vitro revealed mostly typical spindle-shaped fibroblast morphology similar to previously cultured primary murine lung fibroblasts (15) and reminiscent of differentiated peripheral blood-derived fibrocytes (11, 34). Virtually all the $\mathrm{GFP}^{+}$cells from fibrotic lung 

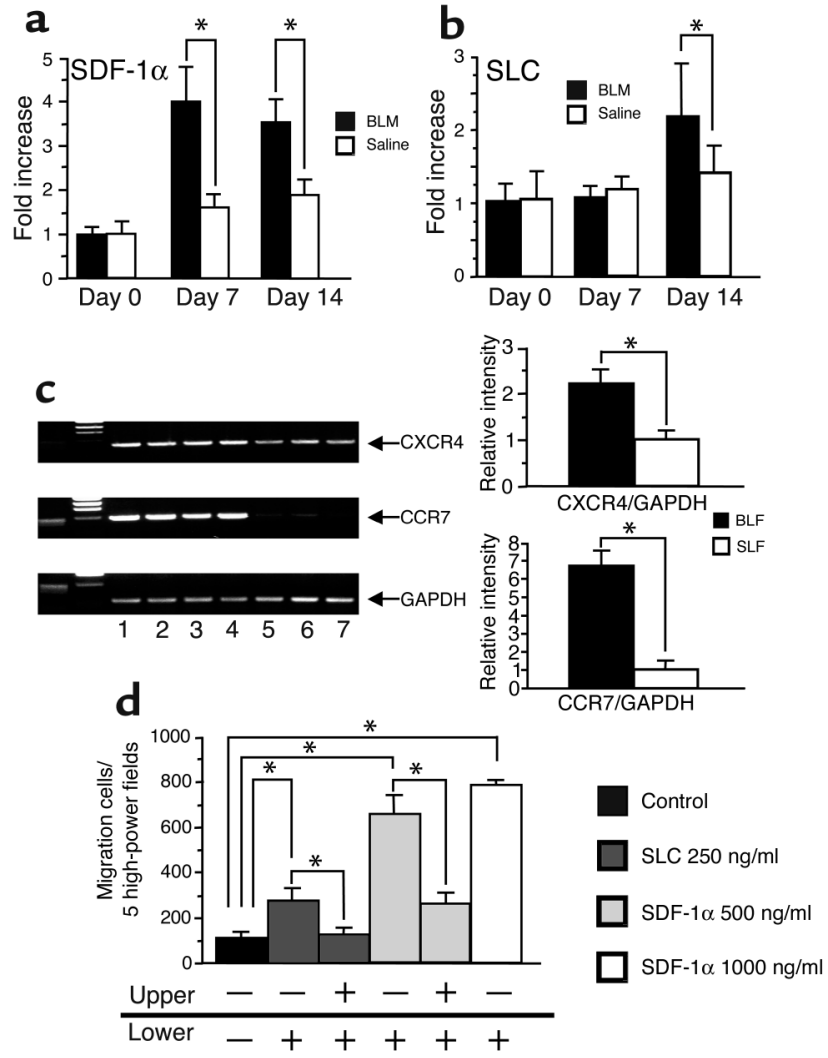

Figure 6

Chemokine and chemokine receptor expression, and fibroblast migration. Lung RNA from saline-treated or BLM-treated mice were obtained at the indicated time points (days after saline or BLM treatment, with day 0 indicating pretreatment values) and analyzed for SDF- $1 \alpha(\mathbf{a})$ and SLC (b) mRNA using real-time PCR. Data shown at each time point represent the means \pm SD from six BLM-treated or saline-treated mice, respectively, and are representative of two independent experiments. (c) Results of RT-PCR analysis for CXCR4 and CCR7 mRNA in cultured BLF or SLF. The left panel in c shows a representative electropherogram of the indicated products using RNA samples from: spleen (lane 1), BLF (lanes 2-4), and SLF (lanes 5-7). The right panel in c summarizes the quantitative results after normalization to the GAPDH signal. Data shown represent the means \pm $\mathrm{SD}(n=3)$, and are representative of three independent experiments. BLF were analyzed for migratory activity toward the indicated chemokine (d). Additions to the upper (where cells were loaded) or lower chamber were as indicated. Both SLC and SDF-1 $\alpha$ were chemotactic for lung fibroblasts, and SDF- $1 \alpha$ was also weakly chemokinetic. Data shown represent means \pm SD. The experiment was repeated once with similar results. Asterisks signify statistically significant difference $(P<0.05)$ between the two groups indicated by connecting lines above the respective bars.

expressed Col I by dual immunofluorescence, but did not express $\alpha$-SMA, a marker of myofibroblasts, suggesting that the latter were not of BM origin. Fibroblast expression of both $\alpha$-SMA and collagen are regulated by TGF- $\beta$, a key mediator of fibrosis (35-37). However, BMderived fibroblasts were resistant to TGF- $\beta$-induced myofibroblast differentiation. The basis for this lack of response to TGF- $\beta$ is unclear at this time but was not due to the ability of TGF- $\beta$ to inhibit GFP expression, which would give rise to the apparent impression that $\alpha$-SMA expression was induced at the same time that GFP expression was suppressed. Thus myofibroblasts in fibrotic lung appear to be not of BM origin but to be perhaps derived locally (i.e., intrapulmonary origin) from quiescent fibroblasts normally found in the peribronchial and perivascular adventitia as previously suggested (36). This would indicate a basic phenotypic difference between resident lung fibroblasts and those derived from BM progenitor cells.

This difference apparently did not extend to telomerase expression. Induction of telomerase expression in fibroblasts is another phenotypic feature characterizing cells in BLM-induced pulmonary fibrosis (17). TERT is the key component of the telomerase complex that is associated with induction of telomerase activity in adult somatic cells that do not normally express this activity $(17,38)$, such as during its induction in lung injury and fibrosis $(17,39)$. The precise role of telomerase in fibrosis is not clear but may be related to its ability to enhance fibroblast proliferative potential and survival (40). The findings in this study show that, in contrast to $\alpha$-SMA expression, a substantial percentage of the TERT ${ }^{+}$cells were $\mathrm{GFP}^{+}$and thus were derived from $\mathrm{BM}$ precursor cells.

An influx of BM-derived cells into the lung in fibrosis requires that some induced signal in the lung is capable of recruiting these extrapulmonary cells, perhaps along the lines of leukocyte recruitment in pulmonary inflammation. More recently and directly relevant to the BMderived precursor cells, peripheral blood-derived fibrocytes are shown to express chemokine receptors such as CCR7 and CXCR4, and could migrate toward SLC, a CCR7 ligand (11). In this study we demonstrated that the levels of SDF-1 $\alpha$ (CXCR4 ligand) and SLC mRNAs after BLM treatment were significantly higher than those in saline-treated control mice, suggesting that they could serve as the signals for recruitment of BM-derived precursor cells to the injured lung. This possibility was supported by data showing that lung fibroblasts from BLM-treated mice expressed the cognate receptors for both these chemokines, and at a significantly higher level than that seen in cells from saline-treated control mice. To ascertain that these expressed receptors were functional, we showed by an in vitro chemotaxis assay that these cells could chemotactically respond to both SLC and SDF-1 $\alpha$. These findings taken together would be consistent with the possibility that increasing expression of these chemokines in BLM-induced lung fibrosis can lead to migratory recruitment of BM-derived precursor cells into the lung and contribute to the formation of active fibrotic lesions. On the basis of previous studies (41-43), the probable cellular sources for these chemokines are likely to be resident lung fibroblasts, or endothelial and/or epithelial cells.

In summary, we have shown that most collagenexpressing as well as TERT-expressing lung fibroblasts in this model of pulmonary fibrosis were derived from BM precursor cells, perhaps under the influence 
of chemokines such as SDF- $1 \alpha$ and SLC. This finding for the first time directly demonstrates the importance of BM-derived fibroblasts in the pathogenesis of pulmonary fibrosis. Hence, revision of current thinking and approaches to the treatment and management of pulmonary fibrosis, and perhaps fibrosis in other organs, is necessary to accommodate this discovery of the extrapulmonary/BM origin of the fibroblast in fibrosis after due consideration of the known limitations of BLM-induced fibrosis as a model for human pulmonary fibrosis.

\section{Acknowledgments}

We acknowledge the excellent technical assistance of Lisa Riggs. This work was supported in part by grants HL28737, HL31963, and HL52285 from the NIH.

1. Blau, H.M., Brazelton, T.R., and Weimann, J.M. 2001. The evolving concept of a stem cell: entity or function? Cell. 105:829-841.

2. Ferrari, G., et al. 1998. Muscle regeneration by bone marrow-derived myogenic progenitors. Science. 279:1528-1530.

3. Orlic, D., et al. 2001. Bone marrow cells regenerate infarcted myocardium. Nature. 410:701-705.

4. Lagasse, E., et al. 2000. Purified hematopoietic stem cells can differentiate into hepatocytes in vivo. Nat. Med. 6:1229-1234.

5. Krause, D.S., et al. 2001. Multi-organ, multi-lineage engraftment by a single bone marrow-derived stem cell. Cell. 105:369-377.

6. Kotton, D.N., et al. 2001. Bone marrow-derived cells as progenitors of lung alveolar epithelium. Development. 128:5181-5188.

7. Mason, R.J., Schwarz, M.I., Hunninghake, G.W., and Musson, R.A. 1999. NHLBI Workshop Summary. Pharmacological therapy for idiopathic pulmonary fibrosis. Past, present, and future. Am. J. Respir. Crit. Care. Med. 160:1771-1777.

8. Gross, T.J., and Hunninghake, G.W. 2001. Idiopathic pulmonary fibrosis. N. Engl. J. Med. 345:517-525.

9. Sheppard, D. 2001. Pulmonary fibrosis: a cellular overreaction or a failure of communication? J. Clin. Invest. 107:1501-1502.

10. Tremblay, G.M., Jordana, M., Gauldie, J., and Sarnstrand, B. 1995. Fibroblasts as effector cells in fibrosis. In Pulmonary fibrosis. S.H. Phan and R.S. Thrall, editors. Marcel Dekker Inc. New York, New York, USA. 541-577.

11. Abe, R., Donnelly, S.C., Peng, T., Bucala, R., and Metz, C.N. 2001. Peripheral blood fibrocytes: differentiation pathway and migration to wound sites. J. Immunol. 166:7556-7562.

12. Manfra, D.J., et al. 2001. Leukocytes expressing green fluorescent protein as novel reagents for adoptive cell transfer and bone marrow transplantation studies. Am. J. Pathol. 158:41-47.

13. Morshead, C.M., Benveniste, P., Iscove, N.N., and van der Kooy, D. 2002. Hematopoietic competence is a rare property of neural stem cells that may depend on genetic and epigenetic alterations. Nat. Med. 8:268-273.

14. Teshima, T., et al. 2002. Acute graft-versus-host disease does not require alloantigen expression on host epithelium. Nat. Med. 8:575-581.

15. Huaux, F., Liu, T., McGarry, B., Ullenbruch, M., and Phan, S.H. 2003. Dual roles of IL-4 in lung injury and fibrosis. J. Immunol. 170:2083-2092.

16. Toda, Y., et al. 1999. Application of tyramide signal amplification system to immunohistochemistry: a potent method to localize antigens that are not detectable by ordinary method. Pathol. Int. 49:479-483.

17. Liu, T., Nozaki, Y., and Phan, S.H. 2002. Regulation of telomerase activity in rat lung fibroblasts. Am. J. Respir. Cell Mol. Biol. 26:534-540.

18. Schwartz, R.E., et al. 2002. Multipotent adult progenitor cells from bone marrow differentiate into functional hepatocyte-like cells. J. Clin. Invest. 109:1291-1302. doi:10.1172/JCI200215182.

19. Livak, K.J., and Schmittgen, T.D. 2001. Analysis of relative gene expression data using real-time quantitative PCR and the 2(-Delta Delta C(T)) Method. Methods. 25:402-408.

20. Ahmed, F., et al. 2002. GFP expression in the mammary gland for imaging of mammary tumor cells in transgenic mice. Cancer Res. 62:7166-7169

21. Iwano, M., et al. 2002. Evidence that fibroblasts derive from epithelium during tissue fibrosis. J. Clin. Invest. 110:341-350. doi:10.1172/JCI200215518.

22. Kohyama, T., et al. 2001. Prostaglandin E(2) inhibits fibroblast chemotaxis. Am. J. Physiol. Lung Cell Mol. Physiol. 281:L1257-L1263.

23. Hashimoto, N., et al. 2001. Effect of erythromycin on matrix metalloproteinase-9 and cell migration. J. Lab. Clin. Med. 137:176-183.

24. Chambers, R.C., and Laurent, G.J. 1997. Collagens. In The lung scientific foundations. R.G. Crystal, J.B. West, P.J. Barnes, and E.R. Weibel, editors. Lippincott-Raven Publishers. New York, New York, USA. 709-727.

25. Tomasek, J.J., Gabbiani, G., Hinz, B., Chaponnier, C., and Brown, R.A 2002. Myofibroblasts and mechano-regulation of connective tissue remodelling. Nat. Rev. Mol. Cell Biol. 3:349-363.

26. Adawi, A., et al. 1998. Blockade of CD40-CD40 ligand interactions protects against radiation-induced pulmonary inflammation and fibrosis. Clin. Immunol. Immunopathol. 89:222-230.

27. Theise, N.D., et al. 2002. Radiation pneumonitis in mice: a severe injury model for pneumocyte engraftment from bone marrow. Exp. Hematol. 30:1333-1338.

28. Clark, J.G., et al. 1993. NHLBI workshop summary. Idiopathic pneumonia syndrome after bone marrow transplantation. Am. Rev. Respir. Dis. 147:1601-1606.

29. Wu, Y.P., et al. 2000. Distribution and characterization of GFP $(+)$ donor hematogenous cells in Twitcher mice after bone marrow transplantation. Am. J. Pathol. 156:1849-1854.

30. Imasawa, T., et al. 2001. The potential of bone marrow-derived cells to differentiate to glomerular mesangial cells. J. Am. Soc. Nephrol. 12:1401-1409.

31. Grove, J.E., et al. 2002. Marrow-derived cells as vehicles for delivery of gene therapy to pulmonary epithelium. Am. J. Respir. Cell Mol. Biol. 27:645-651.

32. Fine, A., and Goldstein, R.H. 1997. Animal models of pulmonary fibrosis. In The lung scientific foundations. R.G. Crystal, J.B. West, P.J. Barnes, and E.R. Weibel, editors. Lippincott-Raven Publishers. New York, New York, USA. 2525-2536

33. Crystal, R.G., Bitterman, P.B., Rennard, S.I., Hance, A.J., and Keogh, B.A. 1984. Interstitial lung diseases of unknown cause. Disorders characterized by chronic inflammation of the lower respiratory tract (first of two parts). N. Engl. J. Med. 310:154-166.

34. Yang, L., et al. 2002. Peripheral blood fibrocytes from burn patients: identification and quantification of fibrocytes in adherent cells cultured from peripheral blood mononuclear cells. Lab. Invest. 82:1183-1192.

35. Vyalov, S.L., Gabbiani, G., and Kapanci, Y. 1993. Rat alveolar myofibroblasts acquire alpha-smooth muscle actin expression during bleomycin-induced pulmonary fibrosis. Am. J. Pathol. 143:1754-1765.

36. Zhang, K., Rekhter, M.D., Gordon, D., and Phan, S.H. 1994. Myofibroblasts and their role in lung collagen gene expression during pulmonary fibrosis. A combined immunohistochemical and in situ hybridization study. Am. J. Pathol. 145:114-125.

37. Kapanci, Y., Desmouliere, A., Pache, J.C., Redard, M., and Gabbiani, G. 1995. Cytoskeletal protein modulation in pulmonary alveolar myofibroblasts during idiopathic pulmonary fibrosis. Possible role of transforming growth factor beta and tumor necrosis factor alpha. Am. J. Respir. Crit. Care. Med. 152:2163-2169.

38. Misiti, S., et al. 2000. Induction of hTERT expression and telomerase activity by estrogens in human ovary epithelium cells. Mol. Cell Biol. 20:3764-3771.

39. Nozaki, Y., Liu, T., Hatano, K., Gharaee-Kermani, M., and Phan, S.H. 2000. Induction of telomerase activity in fibroblasts from bleomycininjured lungs. Am. J. Respir. Cell Mol. Biol. 23:460-465.

40. Boklan, J., et al. 2002. Limited proliferation and telomere dysfunction following telomerase inhibition in immortal murine fibroblasts. Cancer Res. 62:2104-2114.

41. Nagasawa, T., et al. 1996. Defects of B-cell lymphopoiesis and bone-marrow myelopoiesis in mice lacking the CXC chemokine PBSF/SDF-1. Nature. 382:635-638.

42. Ponomaryov, T., et al. 2000. Induction of the chemokine stromal-derived factor-1 following DNA damage improves human stem cell function. J. Clin. Invest. 106:1331-1339.

43. Itakura, M., et al. 2001. Blockade of secondary lymphoid tissue chemokine exacerbates Propionibacterium acnes-induced acute lung inflammation. J. Immunol. 166:2071-2079. 